\title{
Quantum-dot Carnot engine at maximum power
}

\author{
Massimiliano Esposito \\ Center for Nonlinear Phenomena and Complex Systems, Université Libre de Bruxelles, CP 231, Campus Plaine, \\ B-1050 Brussels, Belgium \\ Ryoichi Kawai \\ Department of Physics, University of Alabama at Birmingham, 1300 University Boulevard, Birmingham, Alabama 35294-1170, USA \\ Katja Lindenberg \\ Department of Chemistry and Biochemistry and BioCircuits Institute, University of California, San Diego, La Jolla, \\ California 92093-0340, USA \\ Christian Van den Broeck \\ Hasselt University, B-3590 Diepenbeek, Belgium \\ (Received 13 January 2010; published 12 April 2010)
}

\begin{abstract}
We evaluate the efficiency at maximum power of a quantum-dot Carnot heat engine. The universal values of the coefficients at the linear and quadratic order in the temperature gradient are reproduced. Curzon-Ahlborn efficiency is recovered in the limit of weak dissipation.
\end{abstract}

DOI: 10.1103/PhysRevE.81.041106

PACS number(s): 05.70.Ln, 05.20.-y

\section{INTRODUCTION}

The purpose of a heat engine is to transform an amount of heat $Q_{h}$, extracted from a hot reservoir at temperature $T_{h}$, into an amount of work $W$. The efficiency $\eta=W / Q_{h}$ for doing so is at most equal to Carnot efficiency: $\eta \leq \eta_{c}$, with $\eta_{c}=1-T_{c} / T_{h}$. Here $T_{c}$ is the temperature of a second, cold reservoir $T_{c} \leq T_{h}$, in which the remaining energy $Q_{h}-W$ is deposited. The equality is reached for reversible operation, implying that the corresponding power output is zero. Curzon and Ahlborn (CA) were among the first to study the question of efficiency at maximum power [1]. By considering a simple modification of the Carnot engine and after applying the so-called endo-reversible approximation (neglecting dissipation in the auxiliary system), they found the following beautiful expression for the efficiency at maximum power: $\eta_{\mathrm{CA}}=1-\sqrt{T_{c} / T_{h}}=1-\sqrt{1-\eta_{c}}=\eta_{c} / 2+\eta_{c}^{2} / 8+\cdots$. While this formula appears to describe rather well the efficiency of actual thermal plants, and is close to the efficiency at maximum power for several model systems, it is neither an exact nor a universal result, and it is neither an upper nor a lower bound. It has therefore come as a surprise that a number of universal predictions can be made about the expansion of the efficiency at maximum power in terms of $\eta_{c}$. In the regime of linear response, i.e., at first order in $\eta_{c}$, it is found that the efficiency at maximum power is at most half of the Carnot efficiency [2]. In other words, the CA efficiency is an upper bound at the level of linear response. The proof was given for systems operating under steady-state conditions. The upper bound is reached for so-called strongly coupled systems, i.e., systems in which the heat flux and the work-producing flux are proportional to each other. More recently, it has been shown that the quadratic coefficient, equal to $1 / 8$, is also universal for strongly coupled systems in the presence of an additional left-right symmetry in the system [3]. Furthermore, the universality of the coefficients is a direct consequence of the time reversibility of the underlying physical laws. The coefficient $1 / 2$ derives from the symmetry of the Onsager matrix. The coefficient $1 / 8$ can be seen as the implication of Onsager symmetry at the level of nonlinear response.

The above universality predictions have been confirmed in a number of steady-state model systems involving classical particles [4], fermions [5], and bosons [6]. Universality has also been observed in variants of the CA model based on Carnot cycles performed in finite time, even though finding the optimal driving protocol maximizing work extraction can be notoriously difficult [7-13]. The connection with the steady-state analysis has been clarified by identifying the Onsager coefficient for a finite-time Carnot cycle in the linear regime [11]. Furthermore, agreement with the universal quadratic coefficient has also been observed in a Carnot cycle based on a (classical) Brownian particle in a harmonic trap [8].

In this paper we provide a full analysis of a thermal engine undergoing a Carnot cycle, with the auxiliary system consisting of a single-level quantum dot that is switched between a hot and a cold reservoir. We show that the efficiency at maximum power is again consistent with the above discussed universality. Furthermore, CA efficiency at maximum power is obtained exactly at all orders in $\eta_{c}$ in the limit of low dissipation, which is distinct but similar to the case considered in the original CA paper [1].

\section{MODEL}

Our heat engine model consists of a single-level quantum dot interacting with a metallic lead through a tunneling junction. The quantum dot is assumed to have a single energy level $\varepsilon$ near the Fermi level of the lead while other levels in the dot do not contribute to the processes described below. The state of the system is specified by the occupation probability $p(t)$ of having an electron in the dot. The lead plays 


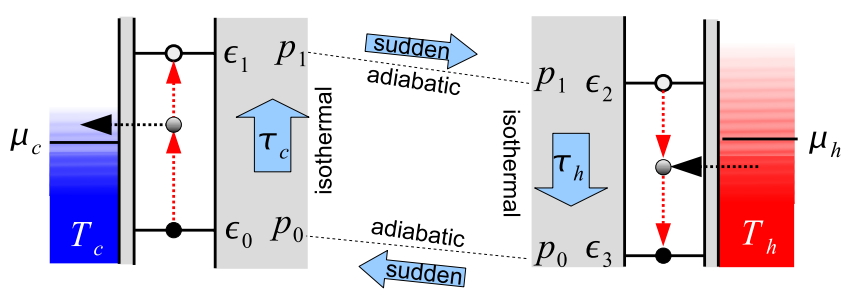

FIG. 1. (Color online) A Carnot cycle of the model heat engine consisting of a single-level quantum dot interacting with a lead through a tunneling junction.

the role of a thermal bath at temperature $T$ and chemical potential $\mu$. Electrons are assumed to thermalize instantaneously upon tunneling into the lead.

When the energy level $\varepsilon$ is modulated by an external agent according to a given protocol, a certain amount of energy, positive or negative, flows into the system in the form of work and/or heat. In the case of an occupied level, an amount of work equal to $\left(\varepsilon_{f}-\mu_{f}\right)-\left(\varepsilon_{i}-\mu_{i}\right)$ is delivered to the system, where the subscripts $f$ and $i$ refer to final and initial values. When the electrons at energy-level $\varepsilon$ tunnel in (out), an amount of heat equal to $Q=\varepsilon-\mu(Q=-\varepsilon+\mu)$ is extracted from the bath.

The basic problem that we address is the finite-time performance of this engine as it runs through the following four standard stages of a Carnot cycle (also see Fig. 1).

\section{A. Isothermal process}

The quantum dot is in contact with a cold lead at temperature $T_{c}$ and chemical potential $\mu_{c}$. The energy level is raised from $\varepsilon_{0}$ to $\varepsilon_{1}$ according to a certain protocol during a time interval of duration $\tau_{c}$. Both work and heat are exchanged during this process.

\section{B. Adiabatic process}

The quantum dot is disconnected from the cold lead, and the quantum level is shifted from $\varepsilon_{1}$ to a new level $\varepsilon_{2}$. Since the quantum dot is thermodynamically isolated, the population of the level does not change during this process. Hence, there is no heat exchange. However, the change of the energy level releases a corresponding amount of work. We assume that the operation time of this step is very short, in particular negligibly small compared to that of the isothermal processes.

\section{Isothermal process}

The dot is connected to the hot lead at temperature $T_{h}$ and chemical potential $\mu_{h}$. The energy level is lowered from $\varepsilon_{2}$ to $\varepsilon_{3}$ based on another protocol during a time interval of length $\tau_{h}$. Just as in step I, both heat and work are exchanged.

\section{Adiabatic process}

The system is again disconnected from the lead and the level is restored from $\varepsilon_{3}$ to the initial level $\varepsilon_{0}$, at the cost of a corresponding amount of work. Afterwards, the dot is re- connected to the cold lead. Again, we assume that the operation time of this process is negligibly small.

The above procedure defines one cycle of the thermal engine, requiring a total time $\tau_{c}+\tau_{h}$. The protocols in steps I and III must be designed in such a way that the thermodynamic state of the system, in our case the occupation probability $p$ of the quantum level, returns to the same initial value after every cycle. Since there is no change in occupation probability during adiabatic stages II and IV, the change in occupation probability from, say $p_{0}$ to $p_{1}$, during process I, must necessarily be compensated by a change back from $p_{1}$ to $p_{0}$ during process III.

The time evolution of the occupation probability $p(t)$ for the state of the quantum dot in contact with a lead at temperature $\beta^{-1}\left(k_{b}=1\right)$ obeys the following quantum master equation:

$$
\dot{p}(t)=-\omega_{a}(t) p(t)+\omega_{b}(t)[1-p(t)],
$$

where the $\omega_{a}$ and $\omega_{b}$ are transition rates. In the wide-band approximation, these rates are given by

$$
\begin{gathered}
\omega_{a}=\frac{C}{e^{-\beta[\varepsilon(t)-\mu(t)]}+1} \\
\omega_{b}=\frac{C}{e^{+\beta[\varepsilon(t)-\mu(t)]}+1},
\end{gathered}
$$

where $C$ is a rate constant. Noting that raising the energy level is equivalent to lowering the chemical potential, we introduce an effective energy level $\epsilon \equiv \varepsilon-\mu$. Master Eq. (1) can now be rewritten as

$$
\dot{p}(t)=-C p(t)+\frac{C}{e^{\beta \epsilon(t)}+1} .
$$

The effective level varies along the Carnot cycle as $\epsilon_{0}=\varepsilon_{0}$ $-\mu_{c} \rightarrow \epsilon_{1}=\varepsilon_{1}-\mu_{c} \rightarrow \epsilon_{2}=\varepsilon_{2}-\mu_{h} \rightarrow \epsilon_{3}=\varepsilon_{3}-\mu_{h}$. Note that the change in the chemical potential is included in the jump of the effective level during processes II and IV.

We next turn to the thermodynamic description of the model. We use the convention that heat entering the system is (like work) positive. The internal energy of the system at time $t$ is

$$
\mathcal{E}(t)=\mathcal{U}(t)-\mu \mathcal{N}(t)=\epsilon(t) p(t),
$$

where

$$
\mathcal{U}(t)=\varepsilon(t) p(t), \quad \mathcal{N}(t)=p(t) .
$$

The rate of change in the internal energy, $\dot{\mathcal{E}}$, is the sum of two parts, namely, a work flux $\dot{\mathcal{W}}$ and a heat flux $\dot{\mathcal{Q}}$,

$$
\begin{aligned}
& \dot{\mathcal{W}} \equiv \dot{\epsilon} p=\dot{\varepsilon} p-\dot{\mu} p \\
& \dot{\mathcal{Q}} \equiv \epsilon \dot{p}=\varepsilon \dot{p}-\mu \dot{p} .
\end{aligned}
$$

Note that the particle exchange contributes to the heat flux [last term in Eq. (6b)]. When the energy level is below the chemical potential, the direction of heat flow is opposite to the direction of tunneling. 
The net total work and net total heat during the process of duration $\tau$ are obtained as functionals of the occupation probability,

$$
\begin{aligned}
& \mathcal{W}[p(\cdot)]=\int_{0}^{\tau} \dot{\epsilon}(t) p(t) d t \\
& \mathcal{Q}[p(\cdot)]=\int_{0}^{\tau} \epsilon(t) \dot{p}(t) d t .
\end{aligned}
$$

For cyclic processes, we have $\mathcal{E}(0)=\mathcal{E}(\tau)$ and hence $\mathcal{W}+\mathcal{Q}$ $=0$. For mathematical simplicity, we evaluate power using net heat instead of net work,

$$
\mathcal{P}=\frac{-\mathcal{W}}{\tau}=\frac{\mathcal{Q}}{\tau} .
$$

\section{OPTIMIZATION: GENERAL CASE}

Our goal is to maximize the power output and to evaluate the corresponding efficiency. Power is a complicated functional of the time-dependent protocols in stages I and III, and an exact analytical analysis looks difficult at first sight. The optimization can however be done in two steps. First, we fix parameter values, $\tau_{c}, \tau_{h}, p_{0}$, and $p_{1}$ and maximize the power with respect to the functional space of $\epsilon(t)$. Since the total operation time is fixed, we just need to maximize the heat. Next, we further maximize the power with respect to the remaining degrees of freedom $\tau_{c}, \tau_{h}, p_{0}$, and $p_{1}$. The problem of maximizing heat or minimizing work for a single-level quantum dot moving between given initial and final energy states has already been analyzed in detail in [14]. We reproduce the crucial steps of this analysis for self-consistency.

To find the protocol that maximizes the heat, we do not search directly for the optimal schedule $\epsilon(t)$, but identify the optimal occupation probability $p(t)$. This is done by expressing $\epsilon(t)$ in terms of $p(t)$ and $\dot{p}(t)$, and rewriting the heat, Eq. (7b), as a functional of $p(t)$ and $\dot{p}(t)$,

$$
\beta \mathcal{Q}[p(\cdot)]=\int_{0}^{\tau} \mathcal{L}(p, \dot{p}) d t
$$

where

$$
\mathcal{L} \equiv \ln \left[\frac{C}{C p(t)+\dot{p}(t)}-1\right] \dot{p}(t) .
$$

The extremum is found via the standard Euler-Lagrange method, leading, after integration, to

$$
\mathcal{L}-\dot{p} \frac{\partial \mathcal{L}}{\partial \dot{p}}=\frac{\dot{p}^{2}}{(C p+\dot{p})[C(1-p)-\dot{p}]}=K .
$$

Here $K$ is the constant of integration. Solving the quadratic equation for $\dot{p}$, we obtain two first-order ordinary differential equations (ODEs),

$$
\frac{\dot{p}}{C}=\frac{K(1-2 p) \mp \sqrt{K^{2}+4 K p(1-p)}}{2(1+K)} .
$$

The upper sign (-) should be used for upward processes in which the quantum level is raised and the lower sign (+) for downward processes. It is worth mentioning a useful symmetry between electrons and holes. We are using the state of an electron, $\boldsymbol{\epsilon}(t)$ and $p(t)$, to describe the state of the system. Instead, we can also use the state of holes, $-\epsilon(t)$ and 1 $-p(t)$. If $p(t)$ is a solution for an upward process, then 1 $-p(t)$ is a solution for a downward process with $-\epsilon(t)$. Hence we do not need to calculate the downward process separately, as it follows from this symmetry.

Before turning to the solution of differential Eq. (12), we examine the physical meaning of the constant $K$. Eliminating $\dot{p}$ in Eq. (11) by using master Eq. (3), the resulting quadratic equation for $p(t)$ leads to the relation

$$
p(t)=\frac{1}{e^{\beta \epsilon(t)}+1}\left[1+e^{\beta \epsilon(t) / 2} \sqrt{K}\right] .
$$

This equation indicates that when $K=0, p(t)$ is the equilibrium distribution associated with the instantaneous value of the energy, implying that $K=0$ corresponds to the quasistatic limit $(\tau \rightarrow \infty)$. As $K$ increases, $p(t)$ deviates from the equilibrium distribution. We conclude that $K$ measures how far the state of the system deviates from the quasistatic limit. We will use this insight below to obtain a perturbative solution for small dissipation by assuming that $K$ is small.

Next we proceed to solve Eq. (12). Separation of the variables $p$ and $t$ leads to the following explicit result for the upward processes,

$$
C t=F[p(t) ; K]-F[p(0) ; K],
$$

where

$$
\begin{aligned}
F(p ; K)= & -\frac{1}{2} \ln p+\frac{1}{\sqrt{K}} \arctan \left[\frac{1-2 p}{\sqrt{K+4 p(1-p)}}\right] \\
& +\frac{1}{2} \ln \left[\frac{2 p+K+\sqrt{K^{2}+4 K p(1-p)}}{2(1-p)+K+\sqrt{K^{2}+4 K p(1-p)}}\right] .
\end{aligned}
$$

For the downward processes, we need to use $F(1-p ; K)$.

The value of $K$ is determined by the boundary conditions

$$
C \tau=F[p(\tau) ; K]-F[p(0) ; K]
$$

Note that $K$ depends solely on the operation time $\tau$, the probabilities $p_{0}$ and $p_{1}$, and the tunneling rate $C$ but not on temperature. Unfortunately, function (15) is quite complicated so we cannot obtain an analytical expression for $K$. In general we need to solve for it numerically. However, an exact perturbative solution is possible (cf. Sec. IV).

Having thus obtained the optimal $p(t)$ with $K$ determined by Eq. (14), we insert this expression in Eq. (7b) to obtain the corresponding maximum heat for the optimal upward processes, 


$$
\begin{aligned}
\beta \mathcal{Q} & =\int_{0}^{\tau} \epsilon(t) \dot{p} d t=\int_{p(0)}^{p(\tau)} \epsilon(p) d p \\
& =\int_{p(0)}^{p(\tau)} d p \ln \left[\frac{2 p(1-p)+K+\sqrt{K^{2}+4 K p(1-p)}}{2 p^{2}}\right] \\
& =\mathcal{S}[p(\tau) ; K]-\mathcal{S}[p(0) ; K]=\Delta \mathcal{S},
\end{aligned}
$$

where

$$
\begin{aligned}
\mathcal{S}(p ; K)= & p \ln \left[\frac{2(1-p) p+K-\sqrt{K^{2}+4 K p(1-p)}}{2 p^{2}}\right] \\
& -\sqrt{K} \arcsin \left[\frac{1-2 p}{\sqrt{K+1}}\right] \\
& -\ln \left[\frac{2(1-p)-K-\sqrt{K^{2}+4 K p(1-p)}}{2}\right] .
\end{aligned}
$$

For the downward processes, $\mathcal{S}(p ; K)$ is replaced by $\mathcal{S}(1-p ; K)$.

Out of equilibrium, $\mathcal{S}$ is different from the system entropy $S(p)=-p \ln p-(1-p) \ln (1-p)$. Indeed, $\Delta \mathcal{S}$ is the entropy flow and is related to the system entropy change $\Delta S$ $=S[p(\tau)]-S[p(0)]$ via the always-positive entropy production $\Delta_{i} S=\Delta S-\Delta \mathcal{S} \geq 0$. It is only in the quasistatic limit, where $K \rightarrow 0$ and thus $\Delta_{i} S=0$, that $\mathcal{S}(p ; K)$ reduces to $S(p)$.

We are now ready to apply the above results to our heat engine. To make the connection with the left/right symmetry required for the universality of the coefficient in the quadratic term, cf. the discussion in the introduction, it will be of interest to consider an asymmetry in the rate constant: we will use the subscripts $C_{c}$ and $C_{h}$ for the rate constant $C$ when in contact with the cold and hot reservoir, respectively. Recalling that processes I and III are upward and downward processes, respectively, boundary condition (16) leads to

$$
\begin{gathered}
C_{c} \tau_{c}=F\left(p_{1} ; K_{c}\right)-F\left(p_{0} ; K_{c}\right) \\
C_{h} \tau_{h}=F\left(1-p_{0} ; K_{h}\right)-F\left(1-p_{1} ; K_{h}\right),
\end{gathered}
$$

which determine the integration constants $K_{c}$ and $K_{h}$, respectively.

Substituting $K_{c}$ and $K_{h}$ into Eq. (17), we obtain the amount of heat that enters the system during processes I and III,

$$
\begin{gathered}
Q_{c}=T_{c}\left[\mathcal{S}\left(p_{1} ; K_{c}\right)-\mathcal{S}\left(p_{0} ; K_{c}\right)\right]=T_{c} \Delta \mathcal{S}_{c} \\
Q_{h}=T_{h}\left[\mathcal{S}\left(1-p_{0} ; K_{h}\right)-\mathcal{S}\left(1-p_{1} ; K_{h}\right)=T_{h} \Delta \mathcal{S}_{h},\right.
\end{gathered}
$$

which leads to the efficiency of the engine

$$
\eta=1+\frac{T_{c} \Delta \mathcal{S}_{c}}{T_{h} \Delta \mathcal{S}_{h}} .
$$

In the quasistatic limit, $K_{c} \rightarrow 0$ and $K_{h} \rightarrow 0$, one has $\mathcal{S}(p ; 0)$ $=S(p)=S(1-p)$, hence $\Delta \mathcal{S}_{c}=-\Delta \mathcal{S}_{h}$ so that Eq. (21) reduces to Carnot efficiency.

The above results provide the required optimization with respect to the schedules. It remains to perform the optimization with respect to the remaining degrees of freedom $\tau_{c}, \tau_{h}$, $p_{0}$, and $p_{1}$. In general, this can only be done numerically since Eq. (14) only provides an implicit equation for the time dependence of the optimal schedule. We are, however, mainly interested in the verification of universal features of efficiency at maximum power. We therefore proceed with a perturbative analysis for which analytic solutions can be obtained.

\section{WEAK DISSIPATION LIMIT}

The deviation from Carnot efficiency can be investigated using the theory of linear irreversible thermodynamics where $T_{h}-T_{c}$ is assumed to be smaller than the temperatures $T_{h}$ and $T_{c}$ of the reservoirs. However, for finite-time thermodynamics, a different kind of expansion, directly related to the irreversibility caused by finite operation time, is more natural. As mentioned in Sec. III, $K$ is a direct measure of the deviation from the quasistatic limit. Hence, it is natural to expand thermodynamic quantities in $K$. Since this is an expansion about the reversible case of zero dissipation, we will refer to this as the limit of weak dissipation.

We expand Eq. (15) in a series in $\sqrt{K}$. The leading term is

$$
F(p ; K)=\frac{\arcsin (1-2 p)}{\sqrt{K}} .
$$

With this approximation, we are able to solve Eq. (19) for $K$ to obtain

$$
\sqrt{K_{\alpha}}=\frac{\left|\phi_{1}-\phi_{0}\right|}{C_{\alpha} \tau_{\alpha}}, \quad(\alpha=c, h)
$$

where

$$
\phi_{i}=\arcsin \left(1-2 p_{i}\right), \quad(i=0,1) .
$$

The present expansion is thus valid under the following condition of weak dissipation:

$$
C_{\alpha} \tau_{\alpha} \gg\left|\phi_{1}-\phi_{0}\right|, \quad(\alpha=c, h) .
$$

Note that it can easily be satisfied in our model since the right-hand side is bounded by $\pi$.

Once we find the value of $K$, the remaining calculation is straightforward. Equation (14) leads to the optimal protocols,

$$
p_{c}(t)=\frac{1}{2}\left[1-\sin \left(\frac{t}{\tau_{c}}\left|\phi_{1}-\phi_{0}\right|+\phi_{0}\right)\right]
$$

$$
p_{h}(t)=\frac{1}{2}\left[1+\sin \left(\frac{t}{\tau_{h}}\left|\phi_{1}-\phi_{0}\right|-\phi_{1}\right)\right] .
$$

Expanding in a Taylor series with respect to $\sqrt{K}$, Eq. (18) is approximated by the two lowest-order terms as

$$
\mathcal{S}(p)=S(p)-\arcsin (1-2 p) \sqrt{K} .
$$

Inserting the value of $K$, we obtain the maximum heat 


$$
\begin{gathered}
Q_{c}^{*}=-T_{c} \Delta S-T_{c} \frac{\left(\phi_{1}-\phi_{0}\right)^{2}}{C_{c} \tau_{c}} \\
Q_{h}^{*}=T_{h} \Delta S-T_{h} \frac{\left(\phi_{1}-\phi_{0}\right)^{2}}{C_{h} \tau_{h}}
\end{gathered}
$$

where $\Delta S=S\left(p_{0}\right)-S\left(p_{1}\right)$ is the reversible entropy change. The second term on the right-hand side is the irreversible heat, which has to be small under condition (25) of weak dissipation. In the quasistatic limit $(\tau \rightarrow \infty)$, the second term vanishes and efficiency (21) reaches the Carnot efficiency, as expected.

When the operation time is too short, the irreversible heat becomes dominant and the net heat becomes negative. Equation (28) indicates that positive power can be obtained only if

$$
\frac{\left(T_{h}-T_{c}\right) \Delta S}{\left(\phi_{1}-\phi_{0}\right)^{2}}>\frac{T_{c}}{C_{c} \tau_{c}}+\frac{T_{h}}{C_{h} \tau_{h}} .
$$

This inequality is consistent with the condition of asymptotic expansion (25) and can thus be satisfied even for a large temperature difference.

So far, we have maximized the power only for the fixed operation times $\tau_{c}$ and $\tau_{h}$ and the boundary values $p_{0}$ and $p_{1}$ of the occupation probabilities. Now we further maximize the power

$$
P=\frac{Q_{c}^{*}+Q_{h}^{*}}{\tau_{c}+\tau_{h}}
$$

with respect to the operation times. It is easy to find that the power is a maximum when

$$
\begin{gathered}
\tau_{c}^{*}=\frac{2\left(\phi_{1}-\phi_{0}\right)^{2} T_{c}\left(1+\sqrt{T_{h} C_{c} / T_{c} C_{h}}\right)}{C_{c} \Delta S\left(T_{h}-T_{c}\right)} \\
\tau_{h}^{*}=\frac{2\left(\phi_{1}-\phi_{0}\right)^{2} T_{h}\left(1+\sqrt{T_{c} C_{h} / T_{h} C_{c}}\right)}{C_{h} \Delta S\left(T_{h}-T_{c}\right)} .
\end{gathered}
$$

This optimization reflects the usual competition with the denominator of the power preferring faster operation whereas the numerator suggests a slower schedule to stay closer to Carnot efficiency.

For the asymptotic expansion to be valid, the optimal operation times must satisfy condition (25) of weak dissipation. That is, for process III the following inequality must be satisfied:

$$
\frac{\left|\phi_{1}-\phi_{0}\right|}{C_{h} \tau_{h}^{*}}=\frac{\Delta S}{2\left|\phi_{1}-\phi_{0}\right|} \frac{T_{h}-T_{c}}{T_{h}\left(1+\sqrt{T_{c} C_{h} / T_{h} C_{c}}\right)} \ll 1 .
$$

This can be achieved in two ways. The first one corresponds to the usual condition for linear irreversible thermodynamics, $\left(T_{h}-T_{c}\right) / T_{h} \ll 1$. The alternative is $\left|\Delta S /\left(\phi_{1}-\phi_{0}\right)\right| \ll 1$. In this limit, our result remains valid even for large temperature differences.

With the optimized operation times [Eq. (31)], the resulting power is written as a function of $p_{0}$ and $p_{1}$,

$$
P=\frac{\left(T_{h}-T_{c}\right)^{2}}{4\left(\sqrt{T_{h} / C_{h}}+\sqrt{T_{c} / C_{c}}\right)^{2}} D\left(p_{0}, p_{1}\right),
$$

where

$$
D\left(p_{0}, p_{1}\right)=\frac{\Delta S^{2}}{\left(\phi_{1}-\phi_{0}\right)^{2}} .
$$

The power reaches its maximum when $D\left(p_{0}, p_{1}\right)$ takes a maximum value, $D_{\max }=0.439$ at $p_{0}=p_{1}=0.0832$ or $p_{0}=p_{1}$ $=0.9168$. At these conditions, optimal operation time (31) and maximum heat (28) both vanish. However, the power remains finite. This final optimization thus leads to a singular and unrealistic situation. We note, however, that since Eq. (34) does not depend on the system parameters, the efficiency does in fact not depend on this final optimization step. Therefore, we proceed to evaluate efficiency without further reference to optimal occupation probabilities.

Using maximum heat (28) and optimal time (31), we finally obtain the following remarkable result for the efficiency at the maximum power:

$$
\begin{aligned}
\eta^{*} & =\frac{\eta_{c}\left(1+\sqrt{C_{h} T_{c} / C_{c} T_{h}}\right)}{2\left(1+\sqrt{C_{h} T_{c} / C_{c} T_{h}}\right)-\eta_{c}}, \\
& =\frac{\eta_{c}}{2}+\frac{\eta_{c}^{2}}{4(1+\sqrt{r})}+\frac{\eta_{c}^{3}}{8(1+\sqrt{r})}+o\left(\eta_{c}^{4}\right)
\end{aligned}
$$

with $r=C_{h} / C_{c}$. When $r=1$, efficiency (35) exactly coincides with the Curzon-Ahlborn efficiency $\eta_{C A}=1-\sqrt{T_{c} / T_{h}}$. Note also that the efficiency is bounded below by $\eta_{c} / 2$ for $C_{h} / C_{c} \rightarrow \infty$ and bounded above by $\eta_{c} /\left(2-\eta_{c}\right)$ for $C_{h} / C_{c}$ $\rightarrow 0$. These limits can be realized without violating the condition of weak dissipation.

\section{DISCUSSION}

We have calculated the efficiency $\eta$ at maximum power of a Carnot cycle with a single-level quantum dot as the operational device. Our calculation is in agreement with known universality properties. In particular, the efficiency at maximum power is equal to half of the Carnot efficiency in the regime of linear response, $\eta=\eta_{c} / 2+\cdots$. In the case of a left/right symmetry, corresponding to equal exchange rate coefficients $C_{h}=C_{c}$ of the dot with the heat reservoirs, the coefficient of the quadratic term is also given by its universal value $1 / 8, \eta=\eta_{c} / 2+\eta_{c}^{2} / 8+\cdots$. However, we need to stress that this result was obtained not by an expansion in $\eta_{c}$ but in the limit of weak dissipation. In fact, this calculation adds a new perspective concerning the occurrence of CurzonAhlborn efficiency itself. Indeed, in the presence of left/right symmetry, the efficiency is actually exactly equal to the CA efficiency $\eta_{C A}=1-\sqrt{1-\eta_{c}}$, in the limit of weak dissipation. This limit is reminiscent of the original derivation of CA efficiency, and is in the present model formally similar to the assumption of a linear conduction law between reservoir and quantum dot. However the concept of weak dissipation is more general. It remains to be explored whether this 
observation implies a wider range of validity of CA efficiency. In particular, it could explain why observed efficiencies at maximum power are not very different from CA efficiency in a wide range of systems under operational conditions far from linear response.

\section{ACKNOWLEDGMENTS}

M.E. is supported by the Belgian Federal Government (IAP project "NOSY"). This research is supported in part by the NSF under Grant No. PHY-0855471.

(2007).

[8] T. Schmiedl and U. Seifert, EPL 81, 20003 (2008).

[9] H. Then and A. Engel, Phys. Rev. E 77, 041105 (2008).

[10] A. Gomez-Marin, T. Schmiedl, and U. Seifert, J. Chem. Phys. 129, 024114 (2008).

[11] Y. Izumida and K. Okuda, Phys. Rev. E 80, 021121 (2009).

[12] Y. Izumida and K. Okuda, Prog. Theor. Phys. 178, 163 (2009).

[13] Y. Izumida and K. Okuda, EPL 83, 60003 (2008).

[14] M. Esposito, R. Kawai, K. Lindenberg, and C. Van den Broeck, EPL 89, 20003 (2010). 